\title{
Evidentista - www.evidentista.org
}

\author{
Derek Richards \\ Editor, Evidence-based Dentistry
}

\begin{abstract}
The Evidentista website, launched in 2006, aims to provide "the best clear, concise, clinical evidence that improves cost-effective care" and "evidence-based dentistry training for clinicians, academics and chief dental officers".

Evidence-Based Dentistry (2007) 8, 25-26. doi:10.1038/sj.ebd.6400475
\end{abstract}

The Evidentista website was established with seed funding from the International Association of Dental Research (IDAR) and the Fulbright Scholar Program. It has been developed by the Pan American Centers for Evidence-Based Dentistry and a number of collaborators, namely the Fulbright Scholar Program, IDAR, Pan American Health Organization, Forsyth Institute, Center for Evidence-Based Dentistry, University of Valparaiso Graduate School of Dentistry, University of Pernambuco Dental School, the Centre for Evidence-Based Dentistry, Oxford, and this journal. To meet the website's aims, a number of resources have been selected that provide high-quality clinical evidence:

- Evidence-based Dentistry journal

- Cochrane Library

- National Institute for Health and Clinical Excellence

- Scottish Intercollegiate Guidelines Network

From these resources, information is distilled in the standardised way, using two lay reviewers followed by two oral health professionals trained in evidence-based dentistry (EBD).

\section{Access}

The website itself is accessed via the front page at www.evidentista.org (Figure1). This page allows the user to select their preferred language from English, Portuguese or Spanish. Next, the main navigation page follows (Figure 2), which is the one that most users will presumably bookmark for future reference. The website currently has two main areas:

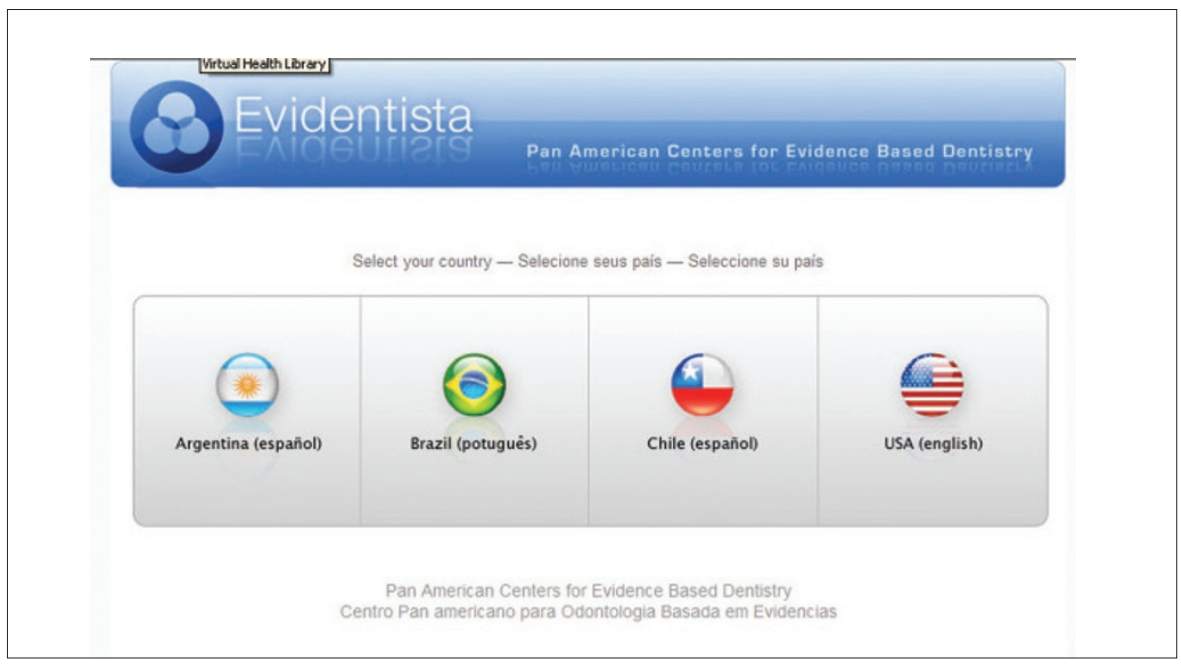

Figure 1. Evidentista front page

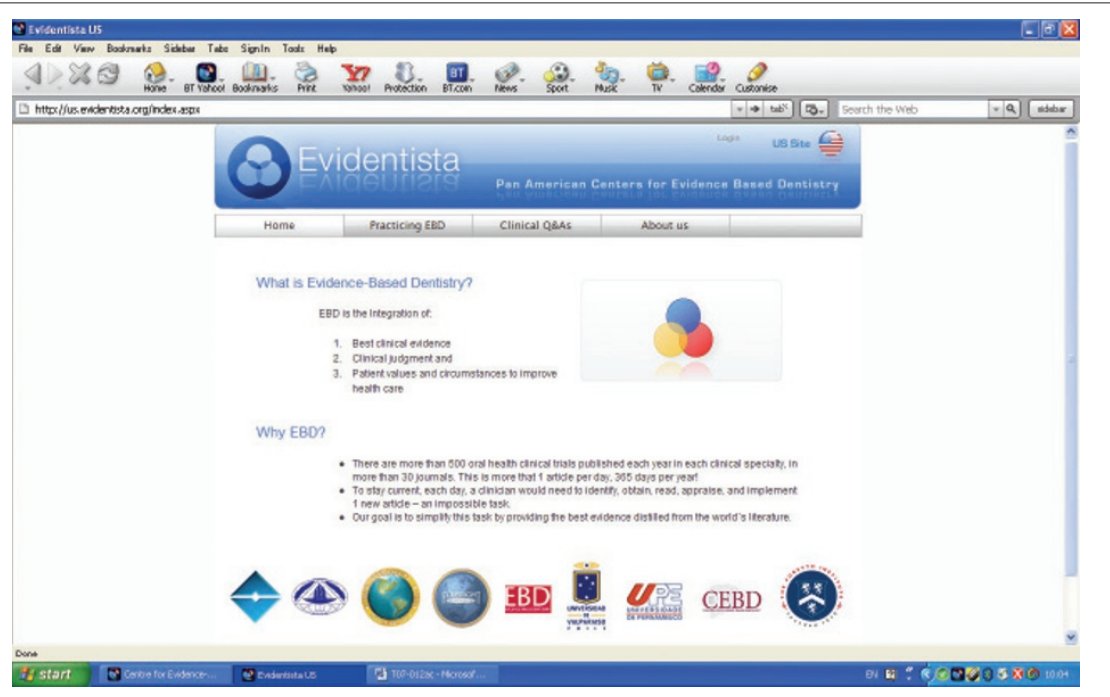

Figure 2. Evidentista main navigation page 
- Practising Evidence-based Dentistry, and

- Clinical Questions and Answers

\section{Practising Evidence-based} Dentistry

These pages briefly outline the five main steps involved in EBD:

1. Asking answerable questions

2. Searching for the best evidence

3. Critically appraising the evidence

4. Applying the evidence

5. Evaluating the outcome

Although the information provided on these pages is not extensive, it is clear and considered. Links are provided to some key resources, although there is scope to improve these.

\section{Clinical questions and answers}

The clinical question and answer section (Figure 3) will, I anticipate, be the major attraction of this site. Currently the site has nine clinical topic areas: clicking on each of these leads to a new page and introduces an additional menu in the lefthand navigation structure. The first element of this menu is Treatment - this is the page presented when "Caries" is selected, for example (Figure 4) - the other elements being Diagnosis, Prognosis and Etiology/ Causation.

On these pages are listed questions, a simple one-word answer (yes, no, unknown), and a brief summary of the main findings with a link to the abstract (Figure 4). Buttons are also provided in order to view the page in the other available languages.

At present there are only a limited number of questions in each section but this will grow in time. This could present the developers with some problems which will need

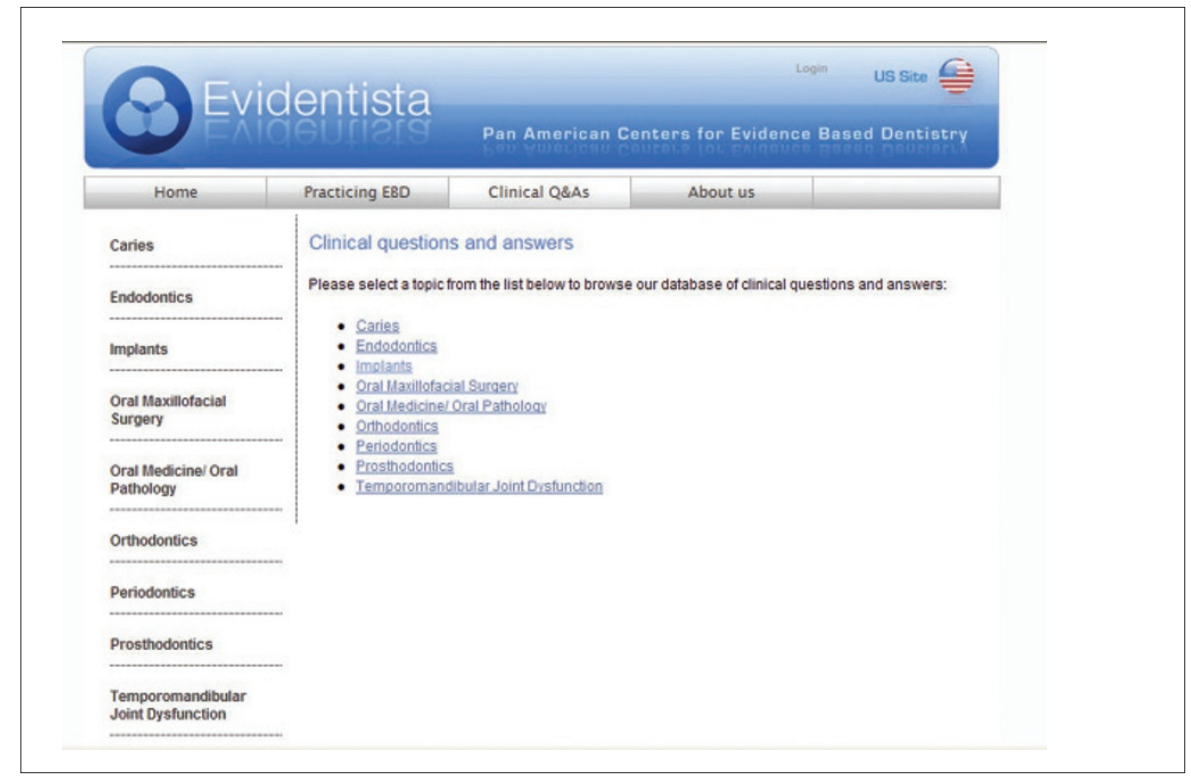

Figure 3. Evidentista Clinical Questions and Answers page

Do topical fluorides effectively reduce caries in children and adolescents?

Yes. Varnish (40\%) had a greater efficacy than rinse (26\%), toothpaste (24\%) and gel $(21 \%)$. (evidence level 1 ). Read the abstract.

\section{Figure 4. Example question and answer} from Evidentista

to be addressed: currently the questions and answers are displayed as a list, which requires the users to scroll through the questions to find what they are looking for - there is no site search engine as yet. There are also some issues regarding the indexing and crossreferencing of questions, for example, the question, "Are resorbable fixation systems as effective as titanium plate systems in orthognathic surgery?" is currently only listed in the orthodontic treatment. I would expect to see it also listed in the oral and maxillofacial surgery treatment section.

These are relatively minor points and I am aware that the developers are seeking support to expand and develop the site. I believe that the website is very useful and helps to address one of the challenges of implementing the evidence. This is the perceived difficulty (usually lack of time and training) in undertaking the second and third stages of the evidence-based approach (searching and appraisal of evidence). Although not addressing the critical appraisal element directly, because only high-quality resources are summarised, the site can provide rapid access to presifted quality information. This will aid the uptake of evidence-based practice. 